\title{
Palliative Care Interventions for Patients with Heart Failure: A Systematic Review and Meta-Analysis
}

\author{
Michelle S. Diop, BA, ${ }^{1,2, *}$ James L. Rudolph, MD, SM, ${ }^{2-4, *}$ Kristin M. Zimmerman, PharmD, CGP, \\ Mary A. Richter, MD, and L. Michal Skarf, MD ${ }^{7,8}$
}

\begin{abstract}
Objective: To systematically characterize interventions and effectiveness of palliative care for advanced heart failure (HF) patients.

Background: Patients with advanced heart failure experience a high burden of distressing symptoms and diminished quality of life. Palliative care expertise with symptom management and healthcare decision-making benefits HF patients.

Methods: A systematic PubMed search was conducted from inception to June 2016 for studies of palliative care interventions for HF patients. Studies of humans with a HF diagnosis who underwent a palliative care intervention were included. Data were extracted on study design, participant characteristics, intervention components, and in three groups of outcomes: patient-centered outcomes, quality-of-death outcomes, and resource utilization. Study characteristics were examined to determine if meta-analysis was possible.

Results: The fifteen identified studies varied in design (prospective, $n=10$; retrospective, $n=5$ ). Studies enrolled older patients, but greater variability was found for race, sex, and marital status. A majority of studies measuring patient-centered outcomes demonstrated improvements including quality of life and satisfaction. Quality-of-death outcomes were mixed with a majority of studies reporting clarification of care preferences, but less improvement in death at home and hospice enrollment. A meta-analysis in three studies found that homebased palliative care consults in HF patients lower the risk of rehospitalization by $42 \%(\mathrm{RR}=0.58 ; 95 \%$ Confidence Interval 0.44, 0.77).

Discussion: Available evidence suggests that home and team-based palliative interventions for HF patients improve patient-centered outcomes, documentation of preferences, and utilization. Increased high quality studies will aid the determination of the most effective palliative care approaches for the HF population.
\end{abstract}

Keywords: cultural care; heart failure; palliative care; quality of life; symptoms; utilization

\section{Introduction}

A DVANCES IN DIAGNOSTIC, pathophysiological, and pharmacological understanding of heart failure (HF) treatments have led to significant improvements in survival. ${ }^{1}$ However, HF incidence and prevalence are increasing, with
HF now affecting more than 5 million Americans. ${ }^{2}$ By age 40 , the lifetime risk of developing HF is 1 in $5 .^{2}$ This translates into extensive morbidity for patients and cost to the healthcare system, with more than a million HF hospital discharges in $2010^{2}$ and an estimated cost of $\$ 32$ billion in 2013. ${ }^{2}$ The American Heart Association projects that by

\footnotetext{
${ }^{1}$ Primary Care and Population Medicine Program, Warren Alpert Medical School of Brown University, Providence, Rhode Island.

${ }^{2}$ Center of Innovation for Long Term Services and Supports, Providence VAMC, Providence, Rhode Island.

${ }^{3}$ Department of Medicine, Warren Alpert Medical School of Brown University, Providence, Rhode Island.

${ }_{5}^{4}$ Center for Gerontology, Brown University School of Public Health, Providence, Rhode Island.

${ }^{5}$ Department of Pharmacotherapy and Outcomes, Virginia Commonwealth University, Richmond, Virginia.

${ }^{6}$ Department of Obstetrics and Gynecology, Tulane University School of Medicine, New Orleans, Louisiana.

${ }^{7}$ Division of Geriatrics and Palliative Care, VA Boston Healthcare System, Boston, Massachusetts.

${ }^{8}$ Harvard Medical School, Boston, Massachusetts.

Accepted October 27, 2016.

*These authors have contributed equally to this work and have agreed to share first authorship.
} 
2030, greater than 8 million Americans will have HF, which will increase the total annual HF cost to $\$ 69.8$ billion. $^{3}$ In addition, death rates remain high; $\sim 50 \%$ of people diagnosed with HF will die within five years, ${ }^{1,2}$ and roughly $5 \%$ of patients have end-stage disease that is refractory to medical therapies. ${ }^{4}$

Care of the HF patient is complex. This care is impacted by a multitude of physical, cognitive, social, and environmental factors. ${ }^{5}$ Disease stage also influences the direction of care. In early HF, medical devices, such as pacemakers and implantable cardioverter defibrillator, are considered beneficial and desired by patients, but this may decline with disease progression. ${ }^{6}$ As HF progresses to the end stage, management of physical, emotional, and spiritual symptoms and advance care planning become the focus of care. Patients with end-stage HF report a high symptom burden, including pain, fatigue, dyspnea, depression, ${ }^{7}$ edema, insomnia, anxiety, anorexia, ${ }^{8}$ confusion, ${ }^{9}$ and a poor overall quality of life. ${ }^{10}$ Prognosis, however, is unpredictable and patients' preferences for care, including quality versus quantity of life, vary. ${ }^{11}$ As a result, a patient-centered approach to advance care planning is imperative. However, recent research indicates a lack of adequate personalized education and communication about HF care. ${ }^{6}$ Considering the complexity of HF care, importance of patient-centered care planning, and lack of communication about treatment options, the utility of palliative care in HF management is fitting. ${ }^{6}$

Studies have demonstrated that the symptom burden associated with HF is comparable to or exceeds that of cancer patients. $^{12}$ Like cancer, HF's high symptom burden includes emotional, spiritual, and caregiver stress as well as uncertain prognosis. Studies of palliative care teams in cancer populations have shown numerous benefits, such as improved quality of life, mood, survival, ${ }^{13}$ pain, and anxiety. ${ }^{14}$ Palliative care has also been shown to decrease costs, ${ }^{15}$ minimize hospitalizations, ${ }^{13,16-19}$ and improve patient satisfaction in a variety of populations. ${ }^{20-25}$ The appropriateness of palliative care and the potential benefit to patients with advanced $\mathrm{HF}$ has been recognized by the American Heart Association (AHA) and the Heart Failure Society of America. However, despite consensus panels and guidelines ${ }^{26,27}$ advocating for the delivery of palliative care concurrent with life-prolonging $\mathrm{HF}$ care, and a recent statement from the AHA recommending referral to palliative care for patients with advanced $\mathrm{HF},{ }^{28}$ there is little consensus regarding specific practices. A systematic review of the impact of palliative care interventions on system process measures and health outcomes in the HF population could drive the design of effective practice recommendations.

It may be hypothesized that including a palliative approach alongside disease-modifying treatment in HF will improve symptom control, leading to decreased hospital admissions, improved mortality, and reduced healthcare costs. The purpose of this article is to summarize the available evidence for palliative care interventions in the HF population and to identify the specific practices that are the most effective in improving patient-centered outcomes, quality of death, and resource utilization. A secondary objective was to examine the available information on demographic and social variability in the available literature.

\section{Methods}

\section{Search methodology}

A systematic search of the PubMed database for peerreviewed English language articles published from inception to June 17, 2016 was conducted by searching for papers that included both an HF term and a palliative care term [see Supplementary Appendix SA1(Supplementary Data are available online at www.liebertpub.com/jpm) for search string]. Titles were reviewed by two authors (M.A.R., M.S.D.), and abstracts meeting eligibility criteria were included for full text review by four authors (M.A.R., L.M.S., M.S.D., J.L.R.). Reference lists were manually searched for additional relevant articles.

\section{Eligibility criteria}

Studies of human subjects with a primary diagnosis of HF who underwent a palliative care or hospice intervention were included. Studies that included other primary diagnoses, such as cancer, cirrhosis, and chronic obstructive pulmonary disease, were included if the study quantified the number of HF participants. All ages and care settings were included. All nonqualitative study designs and outcomes were included. Non-English language articles, studies of a single intervention (e.g., medication for palliation), studies without a comparison group, and studies that did not report the statistical significance of findings were excluded.

\section{Study selection}

Two reviewers (M.A.R., M.S.D.) performed initial independent assessments of titles for relevance. Two additional reviewers agreed on the selection of articles for inclusion (L.M.S., K.Z.), and disputes were adjudicated by a third (J.L.R.). Four reviewers examined abstracts and articles for inclusion (M.A.R., L.M.S., M.S.D., J.L.R.). Bibliographies of full text articles were manually reviewed for additional studies.

\section{Data synthesis}

Data were systematically extracted from articles by using a predefined spreadsheet by two reviewers (M.A.R., M.S.D.) and verified by a second reviewer (L.M.S., J.L.R.). Information extracted included the following: (1) study design, (2) setting, (3) number and characteristics of participants, including HF severity, (4) components of the intervention, (5) comparison group, and (6) outcomes.

\section{Data comparisons}

Palliative care intervention data related to team specialty composition and services offered were abstracted from the selected articles. Data on patient demographics, including age, race, gender, and marital status, were collected to assess selection bias at the study level. Three groups of outcomes were abstracted. Patient-centered outcomes included were patient quality of life, satisfaction, and symptoms. The captured quality-of-death measures included clarification of care preferences, site of death, hospice enrollment, and survival. The resource utilization variables collected were medical service use and cost. Detailed outcomes data were abstracted, as available, from the studies. However, the heterogeneity of the instruments and reporting of these outcomes was complex. 


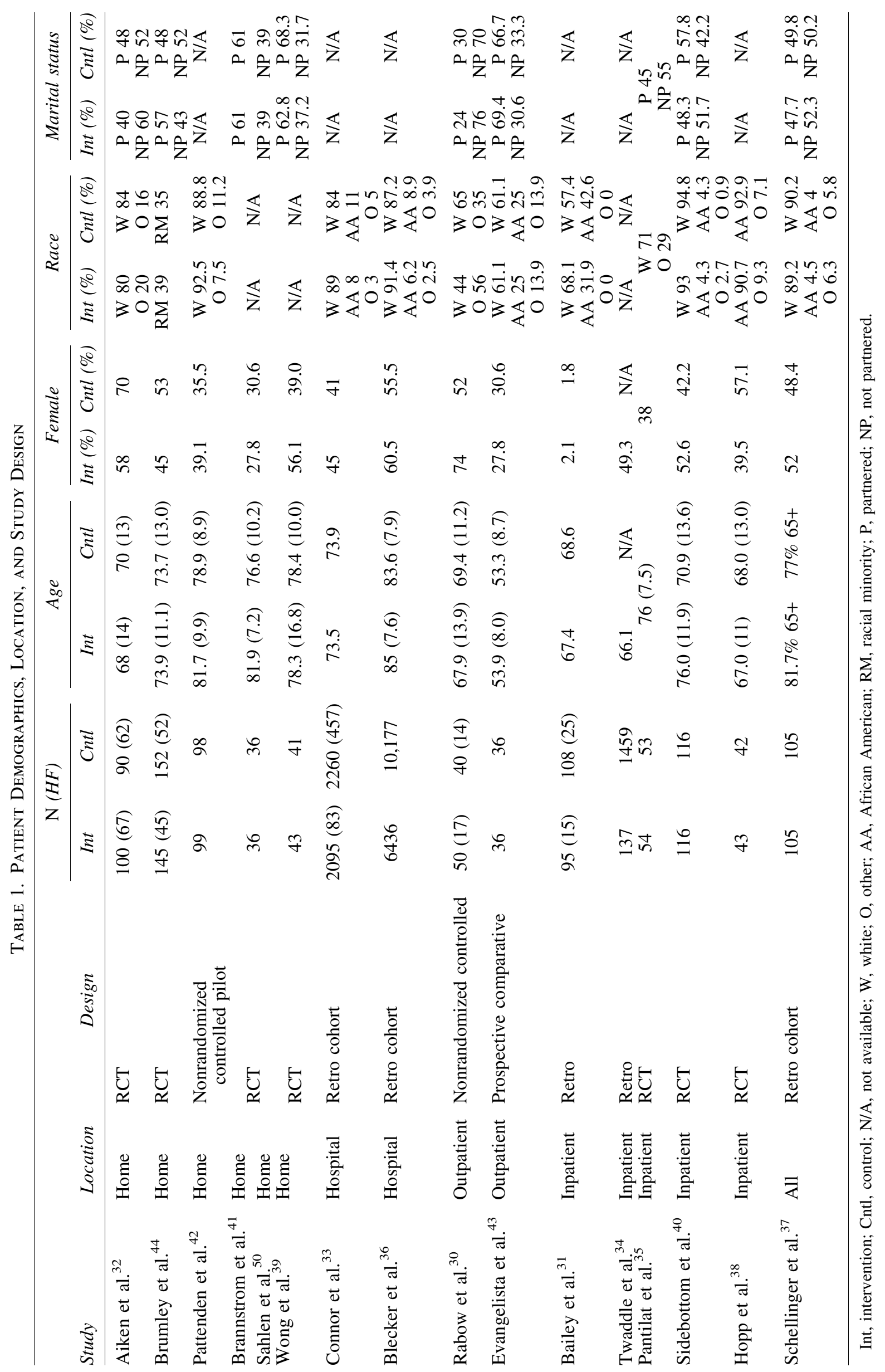




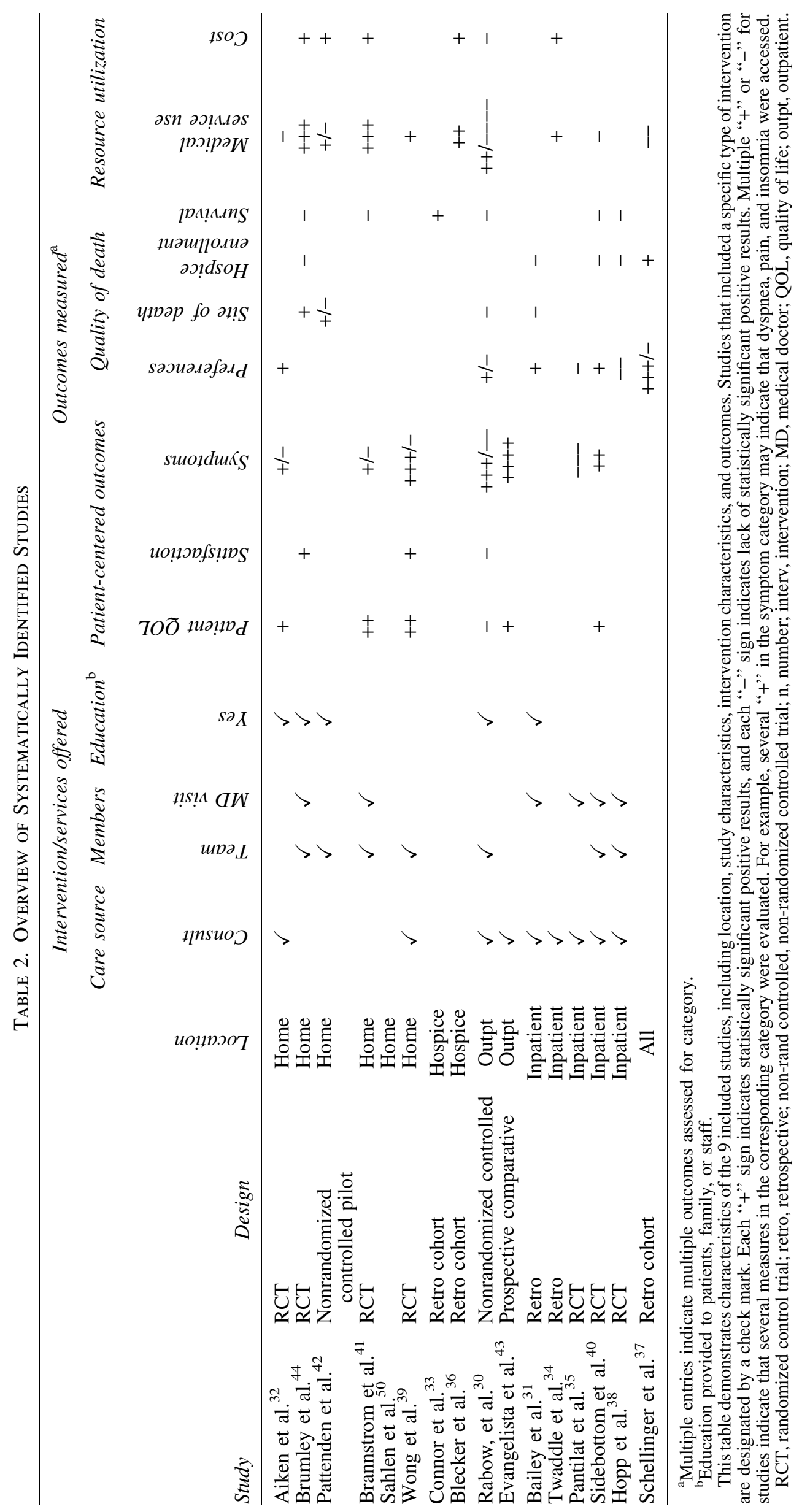




\section{Meta-analysis}

Study location, intervention, and outcomes were examined to determine whether a meta-analysis of outcomes could be performed. Comparison among studies was limited due to heterogeneity and bias in study design, interventions, and outcomes reporting. Stata 11.0 was used to perform a metaanalysis with fixed-effect modeling. Heterogeneity was measured with the $\mathrm{I}^{2}$, an approximation of the variability in the point estimates. ${ }^{29}$ The overall outcome is reported as the relative risk for readmission in those with a palliative care consult with $95 \%$ confidence intervals (CIs).

\section{Results}

Study selection is illustrated in Figure 1. The initial search yielded 1323 studies. Of these, 1197 were excluded based on review of the title. The abstracts of the remaining 126 articles were reviewed. Fifty-five studies were excluded, as they lacked an intervention or comparison group and nine studies were excluded because they were non-English articles. The full texts of the remaining 62 articles were reviewed. Fourteen of the 62 studies met inclusion criteria and were included in the final sample (Fig. 1). ${ }^{30-43}$ A review of bibliographies identified one additional study. ${ }^{44}$ The 15 studies included eight randomized controlled trials, two prospective nonrandomized controlled trials, and five retrospective con- trolled studies. The studies are summarized in Tables 1 and 2 . In Table 2, the number of plus or minus signs corresponds to the numbers of statistically significant or nonsignificant responses, respectively. Supplementary Appendix SA2 includes details on the instrument, magnitude, and significance extrapolated from the studies' tables. More detailed descriptions are available in Supplementary Appendix SA2. In Table 2, the number of plus or minus signs corresponds to the number of statistically significant or nonsignificant responses, respectively.

\section{Participants}

All of the studies included participants with a primary diagnosis of HF. Eight studies included only patients with HF. ${ }^{36-43}$ Other studies included the following additional diagnoses: cancer, ${ }^{30,33-35,44}$ chronic obstructive lung disease, ${ }^{30-32,44}$ cirrhosis, ${ }^{27,35}$ neurologic disease, ${ }^{35}$ and human immunodeficiency virus (HIV). ${ }^{34}$ Study size ranged from 36 patients to 16,613 patients $($ mean $=2629$; median 210$)$. Our review of demographic factors found that studies enrolled older patients, consistent with the pathology of HF. However, there was significant variability with respect to sex and race. Three studies enrolled a majority of women, and five additional studies had one arm with majorities of women. Most studies focused on a white population. However, four studies

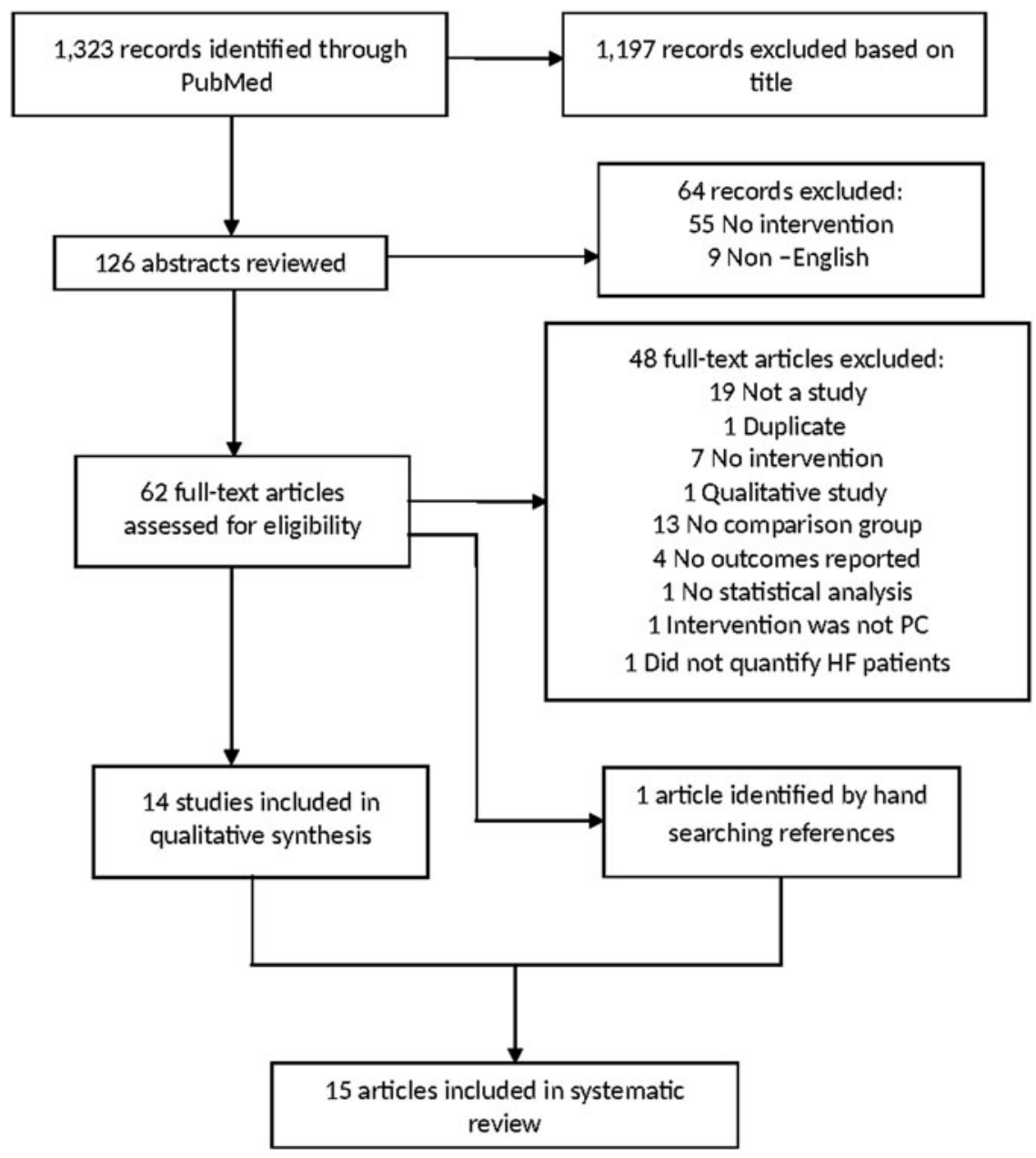

FIG. 1. Flow diagram of study selection process. 
included a more diverse population. Three studies did not include racial demographics, of which two were from nonUS countries and likely did not report race due to homogeneity of the population. Reporting of social factors was also limited. More specifically, there was variability in the participants' marital status, and 6 out of 15 studies did not report on this measure.

\section{Location}

Studies were performed in the following settings: outpatient $(n=2),{ }^{30,43}$ inpatient $(n=5),{ }^{31,34,35,38,40}$ home $(n=5),{ }^{32,39,41,42,44}$ hospice $(n=2),{ }^{33,36}$ and all settings $(n=1)^{37}$ (Table 2$)$.

\section{Interventions}

Interventions varied with respect to team composition and services offered. The studies with improvement in the largest number of outcomes prioritized the integration of interdisciplinary HF and PC care. ${ }^{39,41,42}$ The most common type of intervention utilized a core team of professionals from at least two disciplines with the support of other services as needed, but without emphasizing integrative $\mathrm{PC}$ and $\mathrm{HF}$ care. $^{30,32,37,38,40,44}$ Two interventions were conducted primarily by a physician or nurse. ${ }^{35,43}$ Four studies did not detail a team breakdown. ${ }^{31,33,34,36}$ Initial consults were performed by a physician, ${ }^{31,35,40,41}$ nurse, ${ }^{32,37,39,42}$ social worker, ${ }^{30}$ or full interdisciplinary team. ${ }^{44} \mathrm{~A}$ comprehensive description of services offered and team composition exists in Supplementary Appendix SA2.

\section{Outcomes}

Patient-centered outcomes. Patient quality of life significantly improved in $83 \%$ (5/6) of studies, ${ }^{32,39-41,43}$ and satisfaction significantly improved in $67 \%(2 / 3)$ of studies. $^{30,39,44}$ With respect to symptoms, improvement was the most frequently seen with dyspnea and sleep quality, ${ }^{30,32,39,40,43}$ depression, ${ }^{39,40,43}$ and anxiety. ${ }^{30,32,40}$ The assessment(s) used to gather information about symptoms varied across studies. Tools that assessed a range of symptoms included the Edmonton Symptom Assessment Scale $\left(\mathrm{ESAS}^{45}\right)$ and Kansas City Cardiomyopathy Questionnaire (KCCQ), ${ }^{46}$ but other assessments, such as the Profile of Mood States $^{47}$ and Brief Pain Inventory, ${ }^{48}$ measured individual symptoms. The survey for quality of life also varied across studies. Assessments utilized included the Chronic Heart Failure Questionnaire $\left(\mathrm{CHQ}^{49}\right)$ and Minnesota Living with Heart Failure Questionnaire (MLHF-Q). ${ }^{49}$ The usage of multiple instruments reduced the ability to combine studies for a meta-analysis.

Quality of death. There was inconsistency with respect to improvement among quality-of-death variables. Palliative care interventions were associated with increased documentation of preferences for care in $71 \%(5 / 7)$ of studies, specifically documentation of healthcare proxy, ${ }^{33}$ Physician Orders for Life Sustaining Treatment (POLST), ${ }^{33}$ advance directive, ${ }^{32,33,40}$ do not resuscitate (DNR), ${ }^{31}$ and funeral arrangements. ${ }^{30}$ In contrast to documentation of care preferences, other measures of quality of death either showed minimal improvement or were understudied. Palliative care interventions were associated with an increase in death at

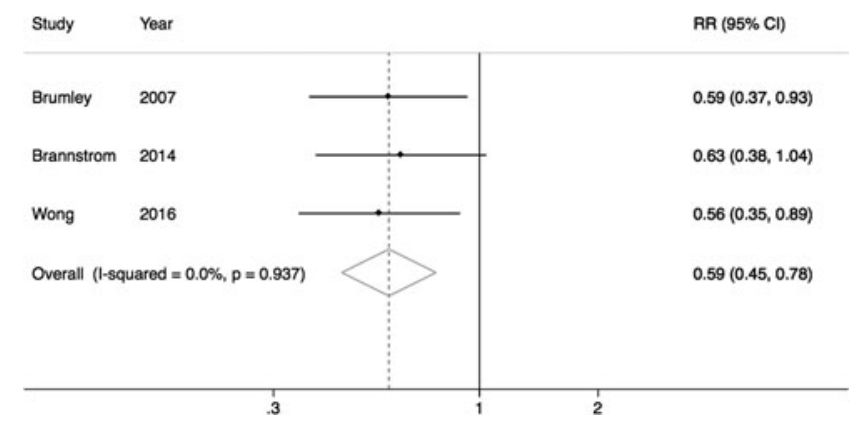

FIG. 2. Meta-analysis of readmissions.

home when compared with the hospital in $50 \%$ of studies (2/4). ${ }^{42,44}$ Hospice enrollment showed a statistically significant increase in only $20 \%(1 / 5)$ of studies. ${ }^{31,37}$ Increased survival was noted in only $17 \%$ of studies measured (1/6), with a survival benefit of 81 days for patients with palliative care. ${ }^{33}$

Resource utilization. Medical service use was the most widely reported variable and showed the greatest improvement. Palliative care interventions were associated with a statistically significant decrease in medical service use in $70 \%(7 / 10)$ of studies. Results were the most robust for decreases in emergency department visits, ${ }^{44}$ length of stay, ${ }^{34,41,44}$ overall admissions, ${ }^{36,39,41,42,44}$ intensive care unit admissions, ${ }^{36}$ urgent care, ${ }^{30}$ and primary care visits. ${ }^{30}$ In our meta-analysis of rehospitalization in three studies, homebased palliative care consults in HF patients lowered the risk of rehospitalization by $42 \%$ (RR $0.5895 \%$ CI [0.44, 0.77]) (Fig. 2). There was no heterogeneity in the population $\left(\mathrm{I}^{2}=0.0 \% ; p=0.91\right)$. The decrease in medical service use was likely of benefit to the patient and was associated with a significant decrease in overall cost of care, as demonstrated in $83 \%(5 / 6)$ of studies. ${ }^{34,36,42,44,50}$

\section{Discussion}

Since management of advanced HF shares common strategies with palliative care, we performed a systematic review to assess the effectiveness of palliative interventions in advanced HF. The systematic search on PubMed identified a growing body of literature to support palliative interventions in HF. Our inclusion criteria were broad, resulting in heterogeneity among the studies selected for inclusion. The finding of reduced rehospitalization among home-based palliative interventions for HF patients suggests an opportunity for reducing transitions in an integrated health system. The review found significant improvement in multiple system process measures and health outcomes supporting the integration of palliative care service and HF disease management. This article also supports the call for further investigation of such integrative interventions to better understand the key factors that contribute to successful care.

As the health system transforms, palliative care is becoming recognized as a critical component of care. This article demonstrates that there is an increasing body of literature focused on the integration of palliative care in $\mathrm{HF}$ management. In this article, we have summarized the existing interventions that may aid palliative care teams in advocating for increased resources to match the needs of our aging 
population. The more recent studies are increasingly focused on examining patient-centered outcomes such as symptoms and quality of life and show positive outcomes in these areas. A need still exists, however, to address the link between social determinants of health and patient-centered outcomes when palliative care is applied to the HF population.

The progressive decline in HF is a sobering and intensely personal experience. During this time, a patient would rely on social coping skills derived from their development and current social environment. As a result, there is a need to provide culturally sensitive care, which involves understanding the social environment of the patient. Although we abstracted measures of this environment by assessing demographic and social variables such as age, race, gender, and partner status, these are crude measures that do not capture the full social environment. Furthermore, some studies failed to report these measures, as evidenced by our finding that $40 \%$ of studies lacked information about the patient's partner status. Recent research, however, suggests that including family caregivers in the disease management of patients with HF may improve outcomes. ${ }^{51}$ In this article, the one study that reported no significant improvement in attitudes toward DNR and hospice enrollment ${ }^{38}$ highlighted a social discordance among the limitations: that the patient population was predominantly African American whereas the providers were mostly Caucasian. The authors recommended that future studies should include more diverse palliative care teams. This is an important consideration, as prior work has demonstrated that there is a gap in knowledge regarding the impact of patient-provider race concordance. ${ }^{52}$ Palliative care is a dynamic process impacted by multiple variables; increasing the information that is reported among studies improves the opportunity to enhance and personalize care for patients.

In addition to the social environment, the services offered and timing of palliative care influence outcomes. Although this article demonstrated that home-based collaborative care improves patient outcomes, analysis was limited due to heterogeneity of the interventions. It should be noted that the majority of participants in these studies carried a poor prognosis. Current research, however, encourages early palliative care interventions for HF patients. ${ }^{6}$ This is especially true given the unpredictable disease course. Early involvement of palliative care would help patients receive the appropriate care, thus improving quality of life. ${ }^{6}$ A major challenge to this charge is the availability of palliative care resources. ${ }^{40}$ A recent review on palliative care in $\mathrm{HF}$ suggests that, at the very least, cardiologists should be equipped with basic tenants of palliative care. ${ }^{6}$ Another major barrier is patients' attitudes toward end-of-life care. One study in our review revealed that some $\mathrm{HF}$ patients decline palliative services out of fear that they will no longer be candidates for aggressive treatment options, whereas others simply refuse to talk about advance care planning. ${ }^{38}$ More studies are needed to elucidate the mechanics and impact of early palliative care interventions for the HF population.

This article has strengths and weaknesses that deserve mention. The search, extraction, and writing of the article followed the PRISMA guidelines, lending strength to this work (Supplementary Appendix SA3). The thorough literature search and careful systematic extraction of data by independent reviewers ensured inclusion of all relevant studies. Through categorizing study design, palliative interventions, and outcomes, this summary provides direction for future projects.

The ability to conduct multiple meta-analyses, however, was limited by the heterogeneity of the studies, which differed with regards to methods, populations, interventions, locations, measurement tools, and outcomes measured. In addition, the studies included minimal information on severity of HF, social determinants of health, types of caregiver involvement, and patient attitudes toward care. Thus, we were unable to conduct analyses on the impact of these factors on the effectiveness of palliative care interventions for patients with advanced HF.

The available studies suggest that interventions consisting of home and team-based palliative care consults combined with HF management improve patient outcomes while decreasing cost and utilization. Given the growth of the HF population, it is imperative that healthcare systems adapt to provide patient-centered decision making and care while decreasing cost. Doing so will require a deeper understanding of the interactions of factors affecting palliative care interventions in the HF population. Future studies should consider the impact of the patient's social environment, services offered, and timing of interventions when designing palliative care programs to maximize the efficacy and quality of care delivered to patients living with HF.

\section{Acknowledgments}

The authors are grateful for the support of Paul Bain, PhD, Countway Library of Medicine, and Erika Sevetson, MS, Alpert Medical School, in assisting with the literature search. This work was funded by the Primary Care-Population Medicine program at Warren Alpert Medical School at Brown University (MD). Additional support was provided by NIH T35-AG038027-02 (M.A.R., J.L.R.). Dr. Rudolph is supported by the VA Health Services Research and Development Center of Innovation in Long Term Services and Supports (CIN 13-419) and the VA QUERI-Geriatrics and Extended Care Partnered Evaluation Center for Community Nursing Homes (PEC 15-465). Drs. Skarf and Rudolph are employees of the Veterans Health Administration. The views expressed are those of the authors and do not reflect the official policies of the Veterans Health Administration or U.S. Government.

\section{Author Disclosure Statement}

No competing financial interests exist.

\section{References}

1. Roger VL, Weston SA, Redfield MM, et al.: Trends in heart failure incidence and survival in a community-based population. JAMA 2004;292:344-350.

2. Mozaffarian D, Benjamin EJ, Go AS, et al.: Executive Summary: Heart Disease and Stroke Statistics-2016 Update: A report from the American Heart Association. Circulation 2016;133:447.

3. Heidenreich PA, Albert NM, Allen LA, et al.: Forecasting the impact of heart failure in the United States: A policy statement from the American Heart Association. Circ Heart Fail 2013;6:606-619.

4. Costanzo MR, Mills RM, Wynne J: Characteristics of "Stage D" heart failure: Insights from the Acute Decompensated 
Heart Failure National Registry Longitudinal Module (ADHERE LM). Am Heart J 2008;155:339-347.

5. Wong CY, Chaudhry SI, Desai MM, Krumholz HM: Trends in comorbidity, disability, and polypharmacy in heart failure. Am J Med 2011;124:136-143.

6. Shah AB, Morrissey RP, Baraghoush A, et al.: Failing the failing heart: A review of palliative care in heart failure. Rev Cardiovasc Med 2013;14:41-48.

7. Goodlin SJ: Palliative care for end-stage heart failure. Curr Heart Fail Rep 2005;2:155-160.

8. McCarthy M, Lay M, Addington-Hall J: Dying from heart disease. J R Coll Physicians Lond 1996;30:325-328.

9. Lynn J, Teno JM, Phillips RS, et al.: Perceptions by family members of the dying experience of older and seriously ill patients. SUPPORT Investigators. Study to understand prognoses and preferences for outcomes and risks of treatments. Ann Intern Med 1997;126:97-106.

10. Bekelman DB, Havranek EP, Becker DM, et al.: Symptoms, depression, and quality of life in patients with heart failure. J Card Fail 2007;13:643-648.

11. Dev S, Abernethy AP, Rogers JG, O’Connor CM: Preferences of people with advanced heart failure-A structured narrative literature review to inform decision making in the palliative care setting. Am Heart J 2012;164:313319.e5.

12. Levenson JW, McCarthy EP, Lynn J, et al.: The last six months of life for patients with congestive heart failure. J Am Geriatr Soc 2000;48(5 Suppl):S101-S109.

13. Temel JS, Greer JA, Muzikansky A, et al.: Early palliative care for patients with metastatic non-small-cell lung cancer. N Engl J Med 2010;363:733-742.

14. Higginson IJ, Evans CJ: What is the evidence that palliative care teams improve outcomes for cancer patients and their families? Cancer J 2010;16:423-435.

15. Morrison RS, Penrod JD, Cassel JB, et al.: Cost savings associated with US hospital palliative care consultation programs. Arch Intern Med 2008;168:1783-1790.

16. Smith TJ, Coyne P, Cassel B, et al.: A high-volume specialist palliative care unit and team may reduce in-hospital end-of-life care costs. J Palliat Med 2003;6:699-705.

17. Elsayem A, Swint K, Fisch MJ, et al.: Palliative care inpatient service in a comprehensive cancer center: Clinical and financial outcomes. J Clin Oncol 2004;22:2008-2014.

18. Norton SA, Hogan LA, Holloway RG, et al.: Proactive palliative care in the medical intensive care unit: Effects on length of stay for selected high-risk patients. Crit Care Med 2007;35:1530-1535.

19. Ciemins EL, Blum L, Nunley M, et al.: The economic and clinical impact of an inpatient palliative care consultation service: A multifaceted approach. J Palliat Med 2007;10:1347-1355.

20. Teno JM, Clarridge BR, Casey V, et al.: Family perspectives on end-of-life care at the last place of care. JAMA 2004;291:88-93.

21. Higginson IJ, Finlay IG, Goodwin DM, et al.: Is there evidence that palliative care teams alter end-of-life experiences of patients and their caregivers? J Pain Symptom Manage 2003;25:150-168.

22. Jordhoy MS, Fayers P, Loge JH, et al.: Quality of life in palliative cancer care: Results from a cluster randomized trial. J Clin Oncol 2001;19:3884-3894.

23. Ringdal GI, Jordhoy MS, Kaasa S: Family satisfaction with end-of-life care for cancer patients in a cluster randomized trial. J Pain Symptom Manage 2002;24:53-63.
24. Christakis NA, Iwashyna TJ: The health impact of health care on families: A matched cohort study of hospice use by decedents and mortality outcomes in surviving, widowed spouses. Soc Sci Med 2003;57:465-475.

25. Casarett D, Pickard A, Bailey FA, et al.: Do palliative consultations improve patient outcomes? J Am Geriatr Soc 2008;56:593-599.

26. Goodlin SJ, Hauptman PJ, Arnold R, et al.: Consensus statement: Palliative and supportive care in advanced heart failure. J Card Fail 2004;10:200-209.

27. Heart Failure Society of America: Disease management in heart failure. J Card Fail 2006;12:e58-e69.

28. Allen LA, Stevenson LW, Grady KL, et al.: Decision making in advanced heart failure: A scientific statement from the American Heart Association. Circulation 2012;125:19281952.

29. Higgins JP, Thompson SG: Quantifying heterogeneity in a meta-analysis. Stat Med 2002;21:1539-1558.

30. Rabow MW, Dibble SL, Pantilat SZ, McPhee SJ: The comprehensive care team: A controlled trial of outpatient palliative medicine consultation. Arch Intern Med 2004;164:83-91.

31. Bailey FA, Burgio KL, Woodby LL, et al.: Improving processes of hospital care during the last hours of life. Arch Intern Med 2005;165:1722-1727.

32. Aiken LS, Butner J, Lockhart CA, et al.: Outcome evaluation of a randomized trial of the PhoenixCare intervention: Program of case management and coordinated care for the seriously chronically ill. J Palliat Med 2006;9:111-126.

33. Connor SR, Pyenson B, Fitch K, et al.: Comparing hospice and nonhospice patient survival among patients who die within a three-year window. J Pain Symptom Manage 2007;33:238-246.

34. Twaddle ML, Maxwell TL, Cassel JB, et al.: Palliative care benchmarks from academic medical centers. J Palliat Med 2007;10:86-98.

35. Pantilat SZ, O'Riordan DL, Dibble SL, Landefeld CS: Hospital-based palliative medicine consultation: A randomized controlled trial. Arch Intern Med 2010;170:20382040.

36. Blecker S, Anderson GF, Herbert R, et al.: Hospice care and resource utilization in Medicare beneficiaries with heart failure. Med Care 2011;49:985-991.

37. Schellinger S, Sidebottom A, Briggs L: Disease specific advance care planning for heart failure patients: Implementation in a large health system. J Palliat Med 2011;14:1224-1230.

38. Hopp FP, Zalenski RJ, Waselewsky D, et al.: Results of a hospital-based palliative care intervention for patients with an acute exacerbation of chronic heart failure. J Card Fail 2016 [Epub ahead of print; DOI: 10.1016/j.cardfail.2016.04.00].

39. Wong FK, Ng AY, Lee PH, et al.: Effects of a transitional palliative care model on patients with end-stage heart failure: A randomised controlled trial. Heart 2016;102:1100-1108.

40. Sidebottom AC, Jorgenson A, Richards H, et al.: Inpatient palliative care for patients with acute heart failure: Outcomes from a randomized trial. J Palliat Med 2015;18:134 142.

41. Brannstrom M, Boman K: Effects of person-centred and integrated chronic heart failure and palliative home care. PREFER: A randomized controlled study. Eur J Heart Fail 2014;16:1142-1151.

42. Pattenden JF, Mason AR, Lewin RJ: Collaborative palliative care for advanced heart failure: Outcomes and costs from the 'Better Together' pilot study. BMJ Support Palliat Care 2013;3:69-76. 
43. Evangelista LS, Lombardo D, Malik S, et al.: Examining the effects of an outpatient palliative care consultation on symptom burden, depression, and quality of life in patients with symptomatic heart failure. J Card Fail 2012;18:894 899.

44. Brumley R, Enguidanos S, Jamison P, et al.: Increased satisfaction with care and lower costs: Results of a randomized trial of in-home palliative care. J Am Geriatr Soc 2007;55:993-1000.

45. Bruera E, Kuehn N, Miller MJ, et al.: The Edmonton Symptom Assessment System (ESAS): A simple method for the assessment of palliative care patients. J Palliat Care 1991;7:6-9.

46. Green CP, Porter CB, Bresnahan DR, Spertus JA.: Development and evaluation of the Kansas City Cardiomyopathy Questionnaire: A new health status measure for heart failure. J Am Coll Cardiol 2000;35:1245-1255.

47. Gibson SJ: The measurement of mood states in older adults. J Gerontol B Psychol Sci Soc Sci 1997;52:P167-P174.

48. Keller S, Bann CM, Dodd SL, et al.: Validity of the brief pain inventory for use in documenting the outcomes of patients with noncancer pain. Clin J Pain 2004;20:309-318.
49. Dunderdale K, Thompson DR, Miles JN, et al.: Quality-of-life measurement in chronic heart failure: Do we take account of the patient perspective? Eur J Heart Fail 2005;7:572-582.

50. Sahlen KG, Boman K, Brannstrom M: A cost-effectiveness study of person-centered integrated heart failure and palliative home care: Based on a randomized controlled trial. Palliat Med 2016;30:296-302.

51. Toukhsati SR, Hare DL: Towards optimal heart failure care: Couples-oriented strategies to improve patient adherence and health outcomes. Curr Cardiol Rev 2016;12:243-248.

52. Meghani SH, Brooks JM, Gipson-Jones T, et al.: Patientprovider race-concordance: Does it matter in improving minority patients' health outcomes? Ethn Health 2009;14:107130.

Address correspondence to:

James L. Rudolph, MD, SM

VA Providence Healthcare System 830 Chalkstone Avenue Providence, RI 02908

E-mail: james_rudolph@brown.edu 